\title{
EDITORIAL
}

\section{Systemic lupus erythematosus-associated PAH: is targeting inflammation the key to success?}

\author{
E. Foïs*,\# and O. Sitbon ${ }^{\tau,+, \S}$
}

$\mathbf{P}$ ulmonary arterial hypertension (PAH) refers to a spectrum of diseases characterised by a permanent increase in pulmonary vascular resistance leading to right ventricular dysfunction and, ultimately, death [1]. PAH can be idiopathic, familial or associated with a number of conditions or diseases, such as congenital heart disease, portal hypertension, HIV infection, and exposure to toxins and drugs, including appetite suppressants. PAH may also develop in the context of connective tissue disease (CTD), and may complicate the course of systemic sclerosis (SSc), systemic lupus erythematosus (SLE), and mixed connective tissue disease (MCTD) [2,3]. Over the past decade, significant advances in the better understanding of $\mathrm{PAH}$ pathophysiology have led to the development of "specific" therapies that target aberrant molecular signalling pathways. Although these treatments favourably impact clinical status, long-term survival remains poor for patients with $\mathrm{PAH}$, particularly for those with CTD $[2,4]$. In the current issue of the European Respiratory Review, JOHNSON and GRANTON [5] give an overview of the epidemiology, prognostic factors and survival of SSc- and SLEassociated PAH. In this review, important differences between these two forms of $\mathrm{PAH}$ are highlighted.

SSc is the most common cause of PAH among CTDs, and SLE represents the second most prevalent condition of CTDassociated $\mathrm{PAH}$, the latter accounting for $15 \%$ of cases in the French PAH registry [6]. In contrast to SSc, no study has been able to accurately establish the prevalence of PAH in SLE, which is reported in the range $0.5-14 \%$ [7]. Such a wide variation reflects the varying definitions of $\mathrm{PAH}$ used, mainly due to differences in investigative modalities employed to establish the diagnosis. In this regard, a major weakness in most studies is the absence of confirmatory right heart catheterisation. In addition, other causes of pulmonary hypertension are not uncommon in SLE patients, highlighting the importance of properly investigating these patients. For example, chronic thromboembolism due to the presence of antiphospholipid antibodies, and diffuse interstitial

\footnotetext{
*Service de Médecine Interne, Hôpital Delafontaine, Saint-Denis, " AP-HP, Service de Médecine Interne, Hôpital Cochin, Paris, "Université Paris-Sud 11, Faculté de Médecine, Le Kremlin-Bicêtre, ${ }^{+}$AP-HP, Centre de Rétérence de l'Hypertension Pulmonaire Sévère, Service de Pneumologie et

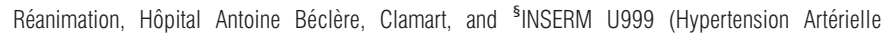
Pulmonaire, Physiopathologie et Innovation Thérapeutique), IPSIT, Centre Chirurgical MarieLannelongue, Le Plessis-Robinson, France.
}

CORRESPONDENCE: 0. Sitbon, Service de Pneumologie, Hôpital Antoine Béclère, 157 rue de la Porte de Trivaux, F-92141 Clamart, France. E-mail: olivier.sitbon@abc.aphp.fr

PROVENANCE: Submitted article, peer reviewed. lung disease may occur in patients with SLE, both of which may be complicated by severe pulmonary hypertension [8].

It is widely accepted that inflammatory and/or immune mechanisms contribute to PAH development and progression [9], especially in patients with CTD, as well as in those with PAHassociated HIV infection or schistosomiasis. Macrophage and lymphocyte infiltrates have been found in plexiform lesions from patients with PAH associated with CTD [10]. The presence of antinuclear antibodies, rheumatoid factor, immunoglobulin $\mathrm{G}$, complement fractions, cytokines and growth factors in pulmonary arteries suggest a role for an immunological mechanism, especially in SLE-associated PAH [11]. These findings strongly support the use of immunosuppressive therapy in patients with CTD-associated PAH. Case reports and small series of patients with PAH associated with SLE or MCTD have highlighted beneficial effects with immunosuppressive therapy, underscoring a possible link between systemic immune dysfunction and pulmonary vasculopathy $[12,13]$. However, no randomised clinical trial has validated use of such agents in this setting. Interestingly, patients with $\mathrm{PAH}$ associated with SSc do not respond to immunosuppressive therapy, suggesting that pathologic changes of pulmonary vessels have already become irreversible in these patients [13]. In contrast, $\mathrm{PAH}$ associated with SLE may respond to a combination of i.v. bolus of cyclophosphamide and systemic glucocorticoids [12, 13]. In the series reported by JAïs et al. [12], 50\% of patients with SLE- or MCTD-associated PAH responded to first-line immunosuppressive therapy alone. Patients with less severe $\mathrm{PAH}$ at the time of diagnosis (New York Heart Association functional class II and preserved cardiac output) are those who may benefit from immunosuppressive therapy; for patients with more severe functional and haemodynamic impairment, $\mathrm{PAH}$-specific agents (i.e. prostanoids and/or endothelin receptor antagonist and/or phosphodiesterase type 5 inhibitor), in combination with immunosuppressive treatment(s) should be considered [12]. Some data suggest that immunosuppressive therapy may be more efficacious in patients with active SLE. However, this strategy may also prove successful in SLE patients with relatively minimal disease activity that have severe PAH. In other words, the presence of none or minimal SLE activity should not preclude the use of immunosuppressive therapy in patients with SLE-associated PAH.

Of considerable interest is the normalisation of haemodynamic parameters in some patients with SLE-associated PAH who received immunosuppressive therapy either alone or in combination with PAH-specific treatment [12, 14, 15]. Indeed, complete 
normalisation of haemodynamic parameters following introduction of PAH-specific therapy in patients with either idiopathic $\mathrm{PAH}$ or $\mathrm{PAH}$ associated with concomitant disease is rare [1]. However, some patients with PAH associated with HIV infection may achieve normalised haemodynamics when a combination of highly active anti-retroviral therapy and PAH-specific therapy is used. Recently, DEGANO et al. [16] reported normalisation of pulmonary haemodynamics in 10 out of 59 patients treated with long-term bosentan in association with anti-retroviral therapy. This also suggests a pivotal role for inflammation in the pathophysiology of certain forms of PAH.

Finally, recent findings suggest that, if appropriately diagnosed and treated, patients with SLE-associated PAH may have a better prognosis than previously suspected, especially in the era of modern PAH-specific therapy [2, 15]. Prospective randomised trials of both immunosuppressive and $\mathrm{PAH}$-specific therapies specifically for patients with SLE-associated PAH are warranted.

\section{STATEMENT OF INTEREST}

O. Sitbon has relationships with drug companies including Actelion, Bayer-Schering, GlaxoSmithKline, Lilly, Pfizer and United Therapeutics. In addition to being an investigator in trials involving these companies, relationships include consultancy service and membership of scientific advisory boards. He has received reimbursement for attending symposium and funds for research from Actelion, Pfizer, GlaxoSmithKline, Lilly and Bayer-Schering. He has recevived reimbursement for attending symposium and funds for research from Actelion, Pfizer, GlaxoSmithKline, Lilly and Bayer-Schering. He has received fees for speaking from Actelion, Bayer-Schering, GlaxoSmithKline, Lilly, Pfizer and United Therapeutics.

\section{REFERENCES}

1 Humbert M, Sitbon O, Simonneau G. Treatment of pulmonary arterial hypertension. N Engl J Med 2004; 351: 1425-1436.

2 Condliffe R, Kiely DG, Peacock AJ, et al. Connective tissue diseaseassociated pulmonary arterial hypertension in the modern treatment era. Am J Respir Crit Care Med 2009; 179: 151-157.
3 Simonneau G, Robbins IM, Beghetti M, et al. Updated clinical classification of pulmonary hypertension. J Am Coll Cardiol 2009; 54: Suppl. 1, S43-S54.

4 Humbert M, Sitbon O, Chaouat A, et al. Survival in patients with idiopathic, familial, and anorexigen-associated pulmonary arterial hypertension in the modern management era. Circulation 2010; 122: $156-163$.

5 Johnson SR, Granton JT. Pulmonary hypertension in systemic sclerosis and systemic lupus erythematosus. Eur Respir Rev 2011; 20: 277-286.

6 Humbert M, Sitbon O, Chaouat A, et al. Pulmonary arterial hypertension in France: results from a national registry. Am J Respir Crit Care Med 2006; 173: 1023-1030.

7 Foïs E, Le Guern V, Dupuy A, et al. Noninvasive assessment of systolic pulmonary artery pressure in systemic lupus erythematosus: retrospective analysis of 93 patients. Clin Exp Rheumatol 2010; 28: 836-841.

8 Torre O, Harari S. Pleural and pulmonary involvement in systemic lupus erythematosus. Presse Med 2011; 40: e41-e51.

9 Dorfmuller P, Perros F, Balabanian $\mathrm{K}$, et al. Inflammation in pulmonary arterial hypertension. Eur Respir J 2003; 22: 358-363.

10 Tuder RM, Groves B, Badesch DB, et al. Exuberant endothelial cell growth and elements of inflammation are present in plexiform lesions of pulmonary hypertension. Am J Pathol 1994; 144: 275-285.

11 Quismorio FP, Sharma O, Koss M, et al. Immunopathologic and clinical studies in pulmonary hypertension associated with systemic lupus erythematosus. Semin Arthritis Rheum 1984; 13: 349-359.

12 Jaïs X, Launay D, Yaïci A, et al. Immunosuppressive therapy in lupus- and mixed connective tissue disease-associated pulmonary arterial hypertension: a retrospective analysis of twenty-three cases. Arthritis Rheum 2008; 58: 521-531.

13 Sanchez O, Sitbon O, Jaïs $X$, et al. Immunosuppressive therapy in connective tissue diseases-associated pulmonary arterial hypertension. Chest 2006; 130: 182-189.

14 Ribeiro JM, Lucas M, Victorino RM. Remission of precapillary pulmonary hypertension in systemic lupus erythematosus. J $R$ Soc Med 2001; 94: 32-33.

15 Heresi GA, Minai OA. Lupus-associated pulmonary hypertension: longterm response to vasoactive therapy. Respir Med 2007; 101: 2099-2107.

16 Degano B, Yaïci A, Le Pavec J, et al. Long-term effects of bosentan in patients with HIV-associated pulmonary arterial hypertension. Eur Respir J 2009; 33: 92-98. 